\title{
In-silico analysis of aging mechanisms of action potential remodeling in human atrial cardiomyocites
}

\author{
Tatyana Nesterova ${ }^{1,2}$, Dmitry Shmarko ${ }^{1,2}$, Konstantin Ushenin ${ }^{1,2}$, and Olga Solovyova ${ }^{1,2, *}$ \\ ${ }^{1}$ Institute of Immunology and Physiology of the Ural Branch of the Russian Academy of Sciences, \\ 620049, Pervomayskaya st., 106, Yekaterinburg, Russia \\ ${ }^{2}$ Ural Federal University named after the first President of Russia B. N. Yeltsin, 620002, Mira st. 19, \\ Yekaterinburg, Russia
}

\begin{abstract}
Electrophysiology of cardiomyocytes changes with aging. Agerelated ionic remodeling in cardiomyocytes may increase the incidence and prevalence of atrial fibrillation (AF) in the elderly and affect the efficiency of antiarrhythmic drugs. There is the deep lack of experimental data on an action potential and transmembrane currents recorded in the healthy human cardiomyocytes of different age. Experimental data in mammals is also incomplete and often contradicting depending on the experimental conditions. In this in-silico study, we used a population of ionic models of human atrial cardiomyocytes to transfer data on the agerelated ionic remodeling in atrial cardiomyocytes from canines and mice to predict possible consequences for human cardiomyocyte activity. Based on experimental data, we analyzes two hypotheses on the aging effect on the ionic currents using two age-related sets of varied model parameters and evaluated corresponding changes in action potential morphology with aging. Using the two populations of aging models, we analyzed the agedependent sensitivity of atrial cardiomyocytes to Dofetilide which is one of the antiarrhythmic drugs widely used in patients with atrial fibrillation.
\end{abstract}

\section{Introduction}

Electrophysiology of cardiomyocytes changes with aging. Age-related changes in the expression level and properties of transmembrane channels and intracellular control pathways affect action potential (AP), transmembrane currents, and balance of intracellular ionic concentrations. The cellular electrophysiological and molecular changes makes the senescent atrial myocardium more susceptible to atrial fibrillation (AF) than that of adults. From the other side, age-related ionic remodeling in cardiomyocytes may change the efficiency of antiarrhythmic drugs.

Experimental data on the effects of antiarrhythmic agents on the human cardiomyocytes of different age is limited. On the other hand, direct translation of the results from animal

\footnotetext{
${ }^{*}$ Corresponding author: soloveva.olga@urfu.ru
} 
studies to the human is doubtful because of significant variability in the data across different species. A population of human ionic models of cardiomyocytes can help for such translation prior to experimental or clinical studies in human.

In this in-silico study, we transfer experimental data on ionic remodeling in aging cardiomyocytes from laboratory animals to simulate population of human atrial cardiomyocytes with advancing age. Here, we used two hypotheses on the combination of ionic currents exposed to age-related modulations. The first hypothesis on age-related modulation in five parameters for three ionic currents $\mathrm{I}_{C a L}, \mathrm{I}_{N a}$ and $\mathrm{I}_{t o}$ was supported by experimental studies in canine atrial cardiomyocytes [1,2]. The second hypothesis assumes an additional modulation of $\mathrm{I}_{K 1}$ current as observed in mice atrial cardiomyocytes [3].

We built two families of aging populations of ionic models of human atrial cardiomyocytes with age-related modulations of each of the two parameter sets according to the experimental data. The ageing populations of models were then experimentally calibrated using rejection criteria for implausible model cases where simulated signals fall out of the ranges of experimental measurements of the AP in human atrial cardiomyocytes.

Then, we used the two families of aging calibrated models to analyze the effects of Dofetilide on the AP across different age groups. Dofetilide was chosen as an antiarrhythmic agent of III class that is widely used for the treatment of AF.

\section{Methods}

In this study, we used the Courtemanche et al. 1998 ([4]) model of the human atrial cardiomyocyte. This model describes cellular AP generation with accounting for twelve transmembrane ionic currents: fast sodium current $\left(\mathrm{I}_{N a}\right)$, L-type calcium current $\left(\mathrm{I}_{C a, L}\right)$, transient outward potassium current $\left(\mathrm{I}_{t o}\right)$, rapid $\left(\mathrm{I}_{K r}\right)$, slow $\left(\mathrm{I}_{K s}\right)$, and ultra-rapid $\left(\mathrm{I}_{K u r}\right)$ delayed rectifier potassium currents, inward rectifier potassium current $\left(\mathrm{I}_{K 1}\right)$, sodiumpotassium $\left(\mathrm{I}_{\mathrm{NaK}}\right)$ and sodium-calcium $\left(\mathrm{I}_{\mathrm{NaCa}}\right)$ exchangers. Also, this model describes intracellular $\mathrm{Ca}^{2+}$ dynamics, including a release and uptake into the sarcoplasmic reticulum.

In our previous work [5], we noticed that the major part of experimental data for building the Courtemanche et al. 1998 model was obtained in patients of 60-62 years old. Thus, reference parameters of the model may be used to simulate cardiomyocyte activity in the human on the border between middle and old age. We denoted this age group here as Senior I group.

Quantitative data on the ionic remodeling in human cardiomyocytes of advancing age is not available. For this reason, we used data on atrial aging in canines and mice. Here, we tested two hypotheses on the age-related changes in parameters of ionic currents. First hypothesis is based on the data from canine $([1,2])$ and assumes age-related progressive change in five parameters of three transmembrane ionic currents: a decrease in the maximal membrane conductance $\mathrm{g}_{C a, L}$ for $I_{C a, L}$, an increase in $\mathrm{g}_{N a}$ for $I_{N a}$ and in $\mathrm{g}_{t o}$ for $I_{t o}$, and nodulation of two kinetic parameters $\tau_{i}$, and $V(0.5)_{i}$ for $I_{t o}$. The set of five parameters is refereed to as $5 \mathrm{P}$ in this paper. Second hypothesis in addition to $5 \mathrm{P}$ takes into account agerelated decrease in the maximal membrane conductance $\mathrm{g}_{K 1}$ for $I_{K 1}$ according to the study [3] in mice. The parameter set is referred to as $5 \mathrm{P}+\mathrm{g}_{K 1}$.

Based on the experimental measurements, we set age-related values of parameters expressed in percentages of the reference values $(100 \%)$ of the Courtemanche model set for the Senior I group. Each combination of age-related parameters (either $5 \mathrm{P}$ or $5 \mathrm{P}+\mathrm{g}_{K 1}$ ) was considered as a 5- or 6-dimensional vector in the varied parameter space. Average values of the age-related parameters in five groups of advancing age from the Adult I group for the young adult age to the Senior III group for the old age are shown in Table 1. An average parameter vector for Adult I group was derived from the experimental data measured in young and old canines $[1,2]$ and mice [3]. Other three average parameter vectors for Adult 
II group of middle age and Senior II, III groups of older age were extrapolated from the linear dependence between parameter vectors for Adult I and Senior I groups. The subpopulations of models for each of the five age group were chosen by random sampling of the parameter vectors within a spherical region of $30 \%$ radius around the average parameter vector for the group specified in Table 1. More detailed description of the sub-population building is presented

$[5]$.

Table 1. Model parameters for aging groups. Two sets of age-related parameters are used for aging populations of models: 5 parameters in upper rows for $5 \mathrm{P}$ set, and additional $\mathrm{g}_{K 1}$ in lower row for $5 \mathrm{P}+\mathrm{g}_{K 1}$ set.

\begin{tabular}{|c|c|c|c|c|c|c|}
\hline & & Adult I & Adult II & Senior I & Senior II & Senior III \\
\hline \multirow[t]{2}{*}{$I C a, L$} & $\mathrm{gCa}, L(\%)$ & 189 & 144.5 & 100 & 55.5 & 11 \\
\hline & $\mathrm{g}_{t o}(\%)$ & 68 & 84 & 100 & 116 & 132 \\
\hline \multirow[t]{2}{*}{ Ito } & $\tau_{i}(\%)$ & 29 & 64.5 & 100 & 135.5 & 171 \\
\hline & $V(0.5)_{i}(\%)$ & 118 & 109 & 100 & 91 & 82 \\
\hline$I N a$ & $\mathrm{~g}_{N a}(\%)$ & 78 & 89 & 100 & 111 & 122 \\
\hline$I_{K 1}$ & $\mathrm{~g}_{K 1}(\%)$ & 176 & 138 & 62 & 24 & \\
\hline
\end{tabular}

We used an open-source software (Myokit [6]) and graphical computational accelerator for model computation. The forward Euler method with a step of $0.005 \mathrm{~ms}$ was used to calculate models during 100 cycles at $1 \mathrm{~Hz}$ pacing rate until approaching the steady-state. The last signals of AP and transmembrane currents in the series were used in the analysis.

Every sub-population of aging models was calibrated using the rejection criteria based on the experimental data to exclude model samples with implausible results. The following criteria were derived from the experimental data [7, 8]: the AP amplitude (APA, $75<$ APA $<137.5 \mathrm{mV}$ ), resting membrane potential (RMP, $-85.2<\mathrm{RMP}<-65 \mathrm{mV}$ ), AP duration (APD) at 20\%, 50\%, 90\% repolarisation $(0.7<$ APD20 < $60 \mathrm{~ms}, 4.9<$ APD50 < $200 \mathrm{~ms}$, $68.9<$ APD90 $<440 \mathrm{~ms})$, membrane potential at the time of $20 \%$ of APD90 $(-35<$ V20 < $10 \mathrm{mV})$.

The rejection of implausible models reduced the size of Adult I and Senior III population by more than $90 \%$ and $60 \%$ respectively. For this reason, we excluded these age groups from the following analysis. The remaining three ageing sub-populations (Adult II containing $1287 / 972$ models in $5 \mathrm{P} / 5 \mathrm{P}+\mathrm{g}_{K 1}$ ) families, respectively; Senior I with $2047 / 1764$ models; Senior II with 2056/1198 models) not only met all calibration criteria for AP characteristics but also agreed with the characteristics of $\mathrm{I}_{\mathrm{Ca}, \mathrm{L}}$ amplitude in human atrial cardiomyocytes [9]. To simulate dofetilide effects in cellular models we used simple poreblock model [10]. The percentage of $I_{K r}$ current inhibition depending on the dofetilide concentration in blood $(C)$ was described using Hill equation:

$$
\mathrm{B}(\%)=\frac{C \mathrm{~h}}{C^{\mathrm{h}}+\mathrm{IC} 50^{\mathrm{h}}}
$$

with $I C 50=8 \mathrm{nM}, h=1$ taken from [11]. The range of unbound effective therapeutic plasma concentrations (ETPC) for dofetilide is $0.4-2 \mathrm{nM}$ [12]. Here, we used three dofetilide concentrations for analysis: $1.25,2.5$, and $5 \mathrm{nM}$. Respectively, $I_{K r}$ was inhibited by $13 \%, 23 \%$ and $38 \%$. Dofetilide of higher concentrations also affects $I_{C a L}, I_{K s}$ and $I_{N a}$ currents, but we have not accounted for these effects in this study. 


\section{Results}

Age-dependent changes in populations of atrial ionic models. Figure 1 shows superposition of AP and $\mathrm{Ca}^{2+}$ transient (CT) signals generated in sub-populations of aging models for the two sets of ionic parameter varied with advancing aging (left and right panels). Independently of the age-dependent parameter set, in Adult II and Senior I groups a spikeand-dome AP morphology is predominant $(93.5 \%$ and $72.8 \%$ for 5P; $96.9 \%$ and $80.9 \%$ for $5 \mathrm{P}+\mathrm{g}_{K 1}$, respectively). For the spike-and-dome AP morphology, the AP recovery phase is not monotonous with characteristic local minimum (spike) during early repolarisation and local maximum (dome) during plateau phases. Unlike the two subpopulations, AP in Senior II group has $100 \%$ models with a triangle AP morphology. Transition from the spike-and-dome to triangle AP shape in advancing age models is due to an increase in $I_{t o}$ current accelerating the early repolarisation, and a decrease in $I_{C a L}$ reducing the AP plateau amplitude in older age groups. In both model families, in concordance with experimental data [13-15] the populations of ageing models reproduced a characteristic reduction in a plateau level V20 with age (Fig. 2, Table 2). The changes in AP morphology in aging populations were accompanied with notable decrease in APD20 and APD50 between the Adult II and Senior II groups (Fig. 2, Table 2). For each age-related parameter set, the populations of aging models demonstrated a reduction in diastolic $\mathrm{Ca}^{2+}$ concentration $\left(\left[\mathrm{Ca}^{2+}\right]_{i, d}\right)$ and CT amplitude (CTA), a reduction in CT duration (CTD) at $50 \%$ peak level (CTD50), but an increase in CTD at 90\% recovery level (CTD90) (Figs. 2, Table 2).
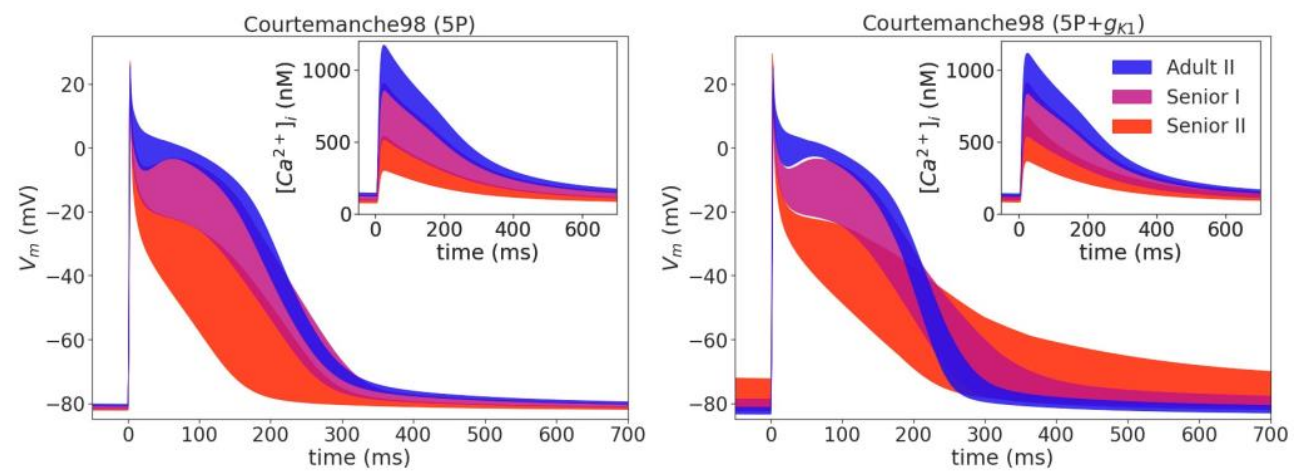

Fig. 1. Action potential (AP) and calcium transients (CT) in the aging populations of models (Adult II, Senior I, and Senior II). Left panel shows simulations at 5P parameter set, and right panel shows results for $5 \mathrm{P}+\mathrm{g}_{K 1}$ parameters.

The distinguishing feature between simulations with $5 \mathrm{P}$ and $5 \mathrm{P}+\mathrm{g}_{K 1}$ parameter sets is that APD90 changes in opposite direction in the two populations with advancing age (Figure 2, Table 2). Namely, APD90 decreases in the older age populations of models in 5P family (average $\triangle \mathrm{APD} 90=-102 \mathrm{~ms}$ or $-35 \%$ in Senior II against Adult II group), while in $5 \mathrm{P}+\mathrm{g}_{K 1}$ family a notable increase in APD90 is shown in Senior II against Adult II group (average $\triangle \mathrm{APD} 90=36 \mathrm{~ms}$ or $14 \%$ ) with increasing variability in APD90 in older age subpopulations ( $\mathrm{STD}$ for $\triangle \mathrm{APD} 90=25 \mathrm{~ms}$ for $5 \mathrm{P}$ and $39.4 \mathrm{~ms}$ for $5 \mathrm{P}+\mathrm{g}_{K 1}$ ). In addition, opposite agedependent changes (less then $5 \%$ ) in the resting membrane potential (RMP) and AP amplitude (APA) are obtained in the populations of models at $5 \mathrm{P}$ and $5 \mathrm{P}+\mathrm{g}_{K 1}$ parameter sets (Figure 2, Table 2).

Dofetilide effects in aging populations of atrial ionic models. In both families of models at $5 \mathrm{P}$ and $5 \mathrm{P}+\mathrm{g}_{K 1}$ parameter sets and in every sub-population of models of different age, Dofetilide increases APD (Figs. 4, 3) in concordance with experimental data [18, 19]. The 
increase in APD was dose-dependent increasing up to $15 \%$ average in Adult II group for for the highest concentration of $5 \mathrm{nM}$ Dofetilid we tested. Increase in APD was accompanied with an increase in CTD, but that was quantitatively less pronounced as compared to the APD change. At the same time, both dialsolic and systolic $\mathrm{Ca}^{2+}$ concentrations $\left(\mathrm{Ca}^{2+}\right]_{i, d}$ and CTA), significantly increase under Dofetilide exposure (Figs. 4, 3). Note, that Dofetilide
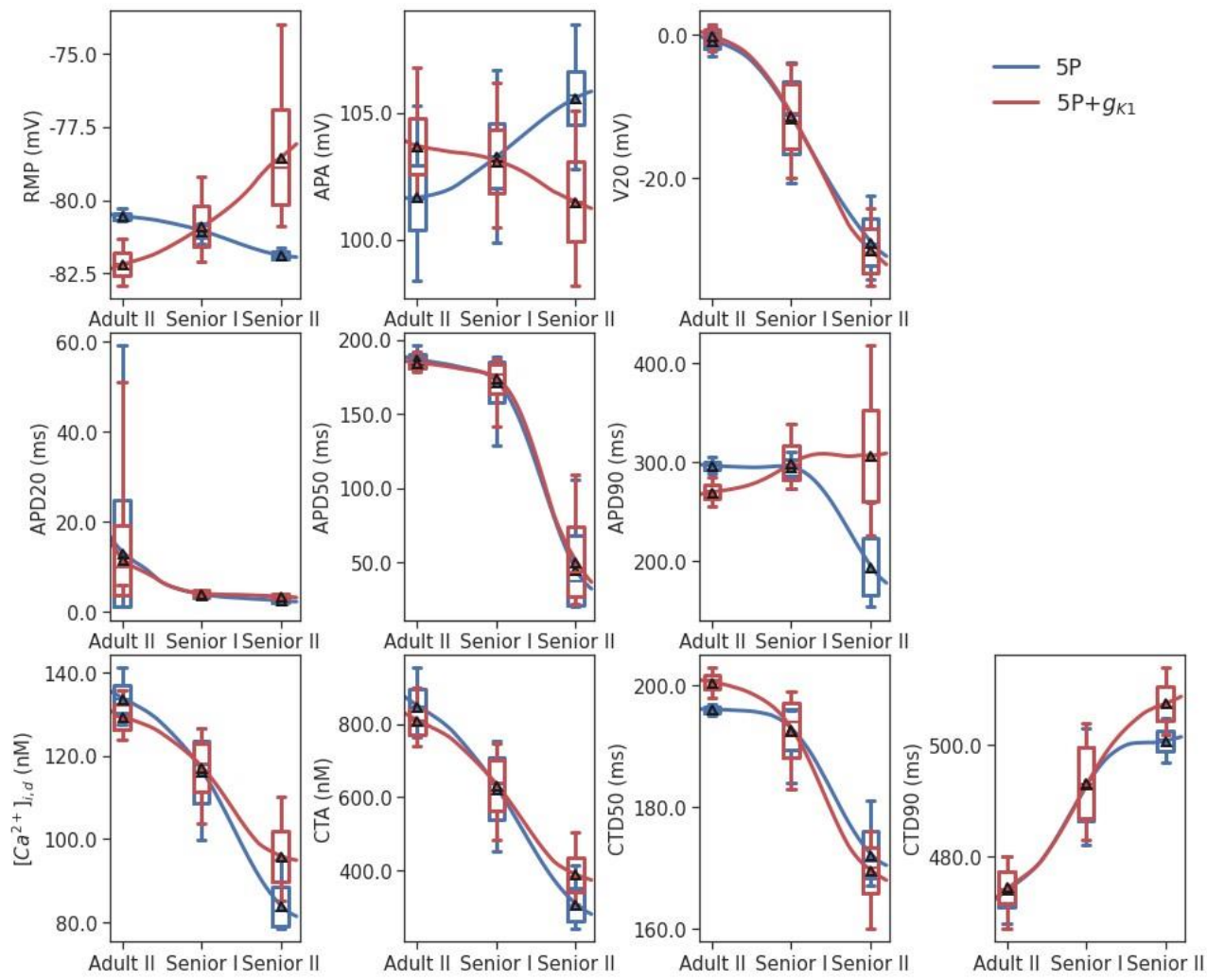

Fig. 2. Age-dependent effects on $\mathrm{AP}$ and $\mathrm{CT}$ features in populations of aging models at $5 \mathrm{P}$ and $5 \mathrm{P}+\mathrm{g}_{K 1}$ parameter sets).

increases frequency of spike-and-dome AP morphology in Adult II and Senior I populations, and increases the plateau level in Senior II population of models (Fig. 3).

In each family of aging models, effect of Dofetilide on APD, and $\mathrm{Ca}^{2+}$ transient were significantly less pronounced in advancing age groups (i.g. average $\triangle$ APD90 about $6 \%$ in Senior II vs $15 \%$ in Adult II group at $5 \mathrm{nM}$ of Dofetilide). Note, that the variance in the response to Defetilide increases in populations of models with advancing age, especially at higher drug concentration.

\section{Discussion}

The major part of available data evidences an age-dependent increase in APD in atrial cardiomyocytes from the mammalian heart of different species (see e.g. data in canines [13-15] and in rabbits ([20]). At the same time there is experimental data showing also insignificant changes in APD90 in left atrium appendage, but APD90 prolongation in the left atrial posterior wall (data in rabbits [21]). There is very limited data showing APD shortening in left atrium, but prolongation in right atrium in rat with advancing age [22]. 
In our simulations, a family of models with $5 \mathrm{P}+\mathrm{g}_{K 1}$ age-related parameter set was able to reproduce an increase in APD90 with age (Fig. 1, 2). This allows us to speculate that $5 \mathrm{P}+\mathrm{g}_{K 1}$ parameter set for the age-related ionic remodeling is more applicable than $5 \mathrm{P}$ parameter set for in-silico studies of aging processes.

Table 2. The difference in AP and CT features between Senior II and Adult II populations of models

\begin{tabular}{c|lll|lll}
\hline & \multicolumn{3}{|c|}{$5 \mathrm{P}$} & \multicolumn{3}{c}{$5 \mathrm{P}+\mathrm{g}_{K 1}$} \\
\hline features & DBM & BHC & MCCBC & DBM & BHC & MCCBC \\
\hline RMP & $-1.34 \mathrm{mV}$ & 0 & 1 & $3.65 \mathrm{mV}$ & 0.01 & 0.98 \\
APA & $3.93 \mathrm{mV}$ & 0.32 & 0.88 & $-2.2 \mathrm{mV}$ & 0.58 & 0.68 \\
V20 & $-28.07 \mathrm{mV}$ & 0 & 1 & $-29.89 \mathrm{mV}$ & 0 & 1 \\
APD20 & $-10.47 \mathrm{~ms}$ & 0 & 1 & $-8.07 \mathrm{~ms}$ & 0 & 1 \\
APD50 & $-141.98 \mathrm{~ms}$ & 0 & 1 & $-134.08 \mathrm{~ms}$ & 0 & 1 \\
APD90 & $-102.44 \mathrm{~ms}$ & 0 & 1 & $36.64 \mathrm{~ms}$ & 0.48 & 0.72 \\
[Ca2+]i,d & $-33.47 \mathrm{nM}$ & 0 & 1 & $-49.71 \mathrm{nM}$ & 0 & 1 \\
CTA & $-538.92 \mathrm{nM}$ & 0 & 1 & $-418.59 \mathrm{nM}$ & 0 & 1 \\
CTD50 & $-23.88 \mathrm{~ms}$ & 0 & 1 & $-30.97 \mathrm{~ms}$ & 0 & 1 \\
CTD90 & $26.61 \mathrm{~ms}$ & 0 & 1 & $33.1 \mathrm{~ms}$ & 0 & 1 \\
\hline
\end{tabular}

Distance between means (DBM); the Bhattacharyya coefficient (BHC) $\in[0,1][16]$ (the less the $\mathrm{BHC}$ the more significant differences between the feature distributions); the Matthews correlation coefficient (MCC) [17] of the Bayesian classifier (MCCBC) $\in[0,1]$ (the more the MCCBC the more significant differences between the feature distributions).
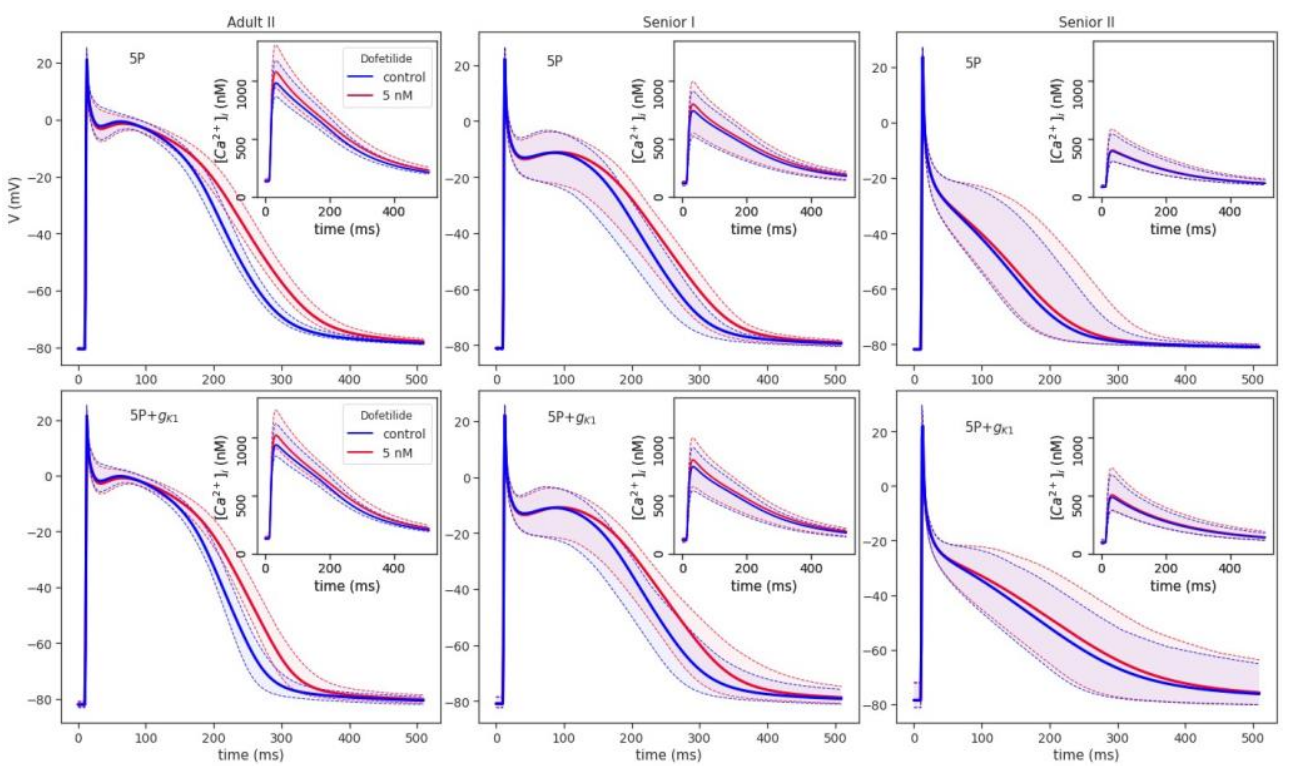

Fig 3. Effect of $5 \mathrm{nM}$ Dofetilide on AP and CT signals in aging populations of models. The upper row shows simulations for $5 \mathrm{P}$ parameter set, and the lower row data is for $5 \mathrm{P}+\mathrm{g}_{K 1}$ family.

There is inconsisting data on the APD50 change in aging cardiomyocytes, showing either an increase or decrease in APD50, or no effect [13, 14, 21]. In our both families of models, APD50 notably decreases with age with much steeper APD50 shortening in transition from Senior I to Senior II group. This could be a prediction for further 
experimental evaluation of the differences between AP for middle age and old age subjects. In both model families, we
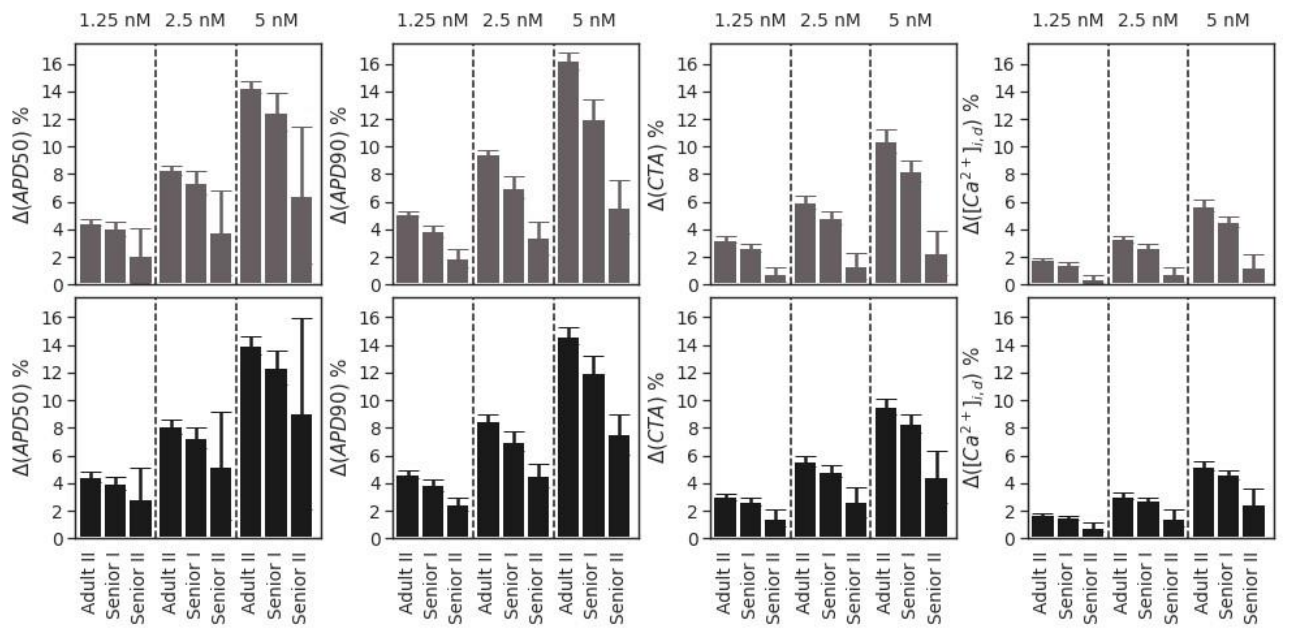

Fig 4. Dose-dependent effect of Dofetilide on AP and CT features in populations of aging models. Three Dofetilide concentrations $(1.25,2.5$, and $5 \mathrm{nM})$ are exposed in three age groups (Adult II, Senior I, and Senior II). Upper row shows results for the 5P family of models, and lower row is for $5 \mathrm{P}+\mathrm{g}_{K 1}$ family.

observed a clear reduction in V20 which is characteristic to senescent atrial AP in canines [13-15]. According to experimental data found no significant changes in RMP and APA values with aging ([13, 15] for RMP and [13, 15, 21] for APA), in our population of models the age-related changes were less than $5 \mathrm{mV}$ in absolute values. However, in several works there are data on statistical significance of APA reduction $([14,21])$ and RMP increase $([14,21])$, which is more consistent again with our simulations generated by $5 \mathrm{P}+\mathrm{g}_{K 1}$ family of models.

Essential decrease in both CTA (more than 50\%) and CTD50 (about 15\%) is shown in the two model families with aging, while CTD90 does not show large age-dependence (about 5\% increase between Adult II and Senior II groups). The decrease in CTA in the populations of models with advancing age is consistent with data in mammalian and human atrial cardiomyocytes [9]. No significant change in CTD50 was observed in experiments in human, that suggests additional parameters of $\mathrm{Ca}^{2+}$ handling should be accounted for in the models to reproduce age-related changes in mechanisms of excitation-contraction coupling. Particularly, an age-related decrease in SERCA pump [9] may contribute to further increase in CTD and APD in the aging populations of models.

Independently of the age-related parameter set, our study shows a reduction in the sensitivity of populations of human atria cardiomyocyte models to Dofetilide with advancing age. A similar result was reported in [23] for canine ventricle cardiomyocytes. Our populations of models show significant increase in APD in response to Dofetilide, especially in Adult II group up to $15 \%$. In resent in-silico study of Dr. B. Rodrigues group [24], an increase in APD by more than 6\% in population of ventricular cardiomyocyte models was shown as a valid predictor of the arrhythogeneity of the drugs. Our model results suggest that both the therapeutic and pro-arrhythmic effects of drugs may change essentially with age, so the use and particular dose of drugs for treatment should be carefully evaluated with accounting for patient age.

Model limitations. In this study, we assessed possible consequences of age-related modulations for two particular sets of ionic parameters in the atrial cells. However, many 
other intracellular mechanisms of excitation-contraction coupling which can be affected by aging were not accounted for. For instance, in article [25] different combination of agerelated changes in $I_{C a L}, I_{K r}, I_{K s}$, and $I_{t o}$ currents is discussed. In article [26] possible consequences of age-related variations in the expression level of Kv4.3, Kv1.4, KChIP2, and KvLQT1 channels are considered. In the article [23] effects of age-related changes in $I_{K s}$ and $I_{K r}$ channels are reported. More comprehensive analysis of the consequences of ionic remodeling in atrial cells with aging should be done in future with accounting for and accurate classification of the exciting experimental data.

\section{Conclusions}

In this work, we build two families of aging populations of the human atrial cardiomyocyte models. The populations are related the different human ages from adult, through middle early senior, to old age. One family of models assumes ionic remodeling with essentially decreasing $\mathrm{I}_{\mathrm{CaL}}$, and increasing $\mathrm{I}_{t o}$ and $\mathrm{I}_{\mathrm{Na}}$ with aging in atrial cardiomyocytes based on data from canine. Second family of models accounts for additional age-related decrease in the $\mathrm{g}_{K 1}$ according to data in mice. Thus, we were trying to translate data from animals to human using in-silico populations of human atrial cells to predict possible consequences of ionic parameter change with age.

According to our results, the ionic parameter remodeling under normal aging process can transfer spike-and-dome AP morphology into triangle shape in atrial cardiomyocytes. Also, the models predict reduction in APD50, V20,CTD50,CTA, $\left[\mathrm{Ca}^{2}+\right]_{i, d}$, and increase in

$C T D 90$. Dispersion of $A P D 50$ and $A P D 90$ increases with age. Changes in $A P D 90$ depend on the combination of varied age-related currents in the populations of models. In our study, accounting for age-related decrease in $\mathrm{g}_{K 1}$ is essential to produce APD90 increase in aging models.

We used the aging populations of models to predict the effects of Dofetilide in the human atrial cardiomyocytes in advancing age. For both model families, we observed a reduction in drug efficiency during the normal cardiomyocyte aging process. An increase in $A P D 90, A P D 50, \mathrm{CTA},\left[\mathrm{Ca}^{2+}\right]_{i, d}$ is stronger in adult age population of models in comparison with senior age populations. Variations in cellular response to Dofetilide increase in older populations of models.

\section{Acknowledgements}

This work was supported by RFBR \#18-015-00368, IIP UrB RAS theme \#AAAAA18118020590031-8, RF Government Act \#211 of March 16, 2013.

\section{References}

1. W. Dun, T. Yagi, M.R. Rosen, P.A. Boyden, Cardiovascular Research, 58, 526 (2003)

2. S. Baba, W. Dun, M. Hirose, P.A. Boyden, American Journal of Physiology-Heart and Circulatory Physiology, 291, H756 (2006)

3. J. Ren, Q. Li, S. Wu, S.Y. Li, S.A. Babcock, Mechanisms of ageing and development, 128, 276 (2007)

4. M. Courtemanche, R.J. Ramirez, S. Nattel, American Journal of Physiology-Heart and Circulatory Physiology , 275, H301 (1998)

5. T. Nesterova, K. Ushenin, D. Shmarko, O. Solovyova, Electrophysiological Biomarkers for Age-Related Changes in Human Atrial Cardiomyocytes: In Silico Study, in ITM Web of Conferences (EDP Sciences, 2020), 31, 01004 
6. M. Clerx, P. Collins, E. de Lange, P.G. Volders, Progress in biophysics and molecular biology, 120, 100 (2016)

7. A. Muszkiewicz, X. Liu, A. Bueno-Orovio, B.A. Lawson, K. Burrage, B. Casadei, B. Rodriguez, American Journal of Physiology-Heart and Circulatory Physiology, 314, H895 (2018)

8. C. Sánchez, A. Bueno-Orovio, E. Wettwer, S. Loose, J. Simon, U. Ravens, E. Pueyo, B. Rodriguez, PloS one, 9 (2014)

9. A. Herraiz-Martínez, J. Álvarez-García, A. Llach, C.E. Molina, J. Fernandes, A. Ferrero-Gregori, C. Rodríguez, A. Vallmitjana, R. Benítez, J.M. Padro et al., Cardiovascular research, 106, 76 (2015)

10. T. Brennan, M. Fink, B. Rodriguez, European Journal of Pharmaceutical Sciences, 36, 62 (2009)

11. H. Sutanto, L. Laudy, M. Clerx, D. Dobrev, H.J. Crijns, J. Heijman, Pharmacological research ,148, 104444 (2019)

12. W. Redfern, L. Carlsson, A. Davis, W. Lynch, I. MacKenzie, S. Palethorpe, P. Siegl, I. Strang, A. Sullivan, R. Wallis et al., Cardiovascular research, 58, 32 (2003)

13. E.P. Anyukhovsky, E.A. Sosunov, A. Plotnikov, R.Z. Gainullin, J.S. Jhang, C.C. Marboe, M.R. Rosen, Cardiovascular research, 54, 462 (2002)

14. E.P. Anyukhovsky, E.A. Sosunov, P. Chandra, T.S. Rosen, P.A. Boyden, P. Danilo Jr, M.R. Rosen, Cardiovascular research, 66, 353 (2005)

15. G.J. Xu, T.Y. Gan, B.P. Tang, Z.H. Chen, A. Mahemuti, T. Jiang, J.G. Song, X. Guo, Y.D. Li, X.H. Zhou et al., Molecular medicine reports, 8, 1343 (2013)

16. T. Kailath, IEEE transactions on communication technology, 15, 52 (1967)

17. B.W. Matthews, Biochimica et Biophysica Acta (BBA)-Protein Structure, 405, 442 (1975)

18. G. Liu, X. Xue, C. Gao, J. Huang, D. Qi, Y. Zhang, J.Z. Dong, C.S. Ma, G.X. Yan, Journal of the American Heart Association, 6, e005482 (2017)

19. C. Martin, M.A. Palomo, E.G. McMahon, J. Pharmacol. Exp. Ther., 278, 154 (1996)

20. N. TODA, Cardiovascular research, 14, 58 (1980)

21. W. Wongcharoen, Y.C. CHEN, Y.J. CHEN, C.I. LIN, S.A. CHEN, Journal of cardiovascular electrophysiology, 18, 526 (2007)

22. C. Huang, W. Ding, L. Li, D. Zhao, Circulation Journal, 70, 352 (2006)

23. M.M.N. Obreztchikova, E.E.A. Sosunov, A. Plotnikov, E.E.P. Anyukhovsky, R.Z. Gainullin, P. Danilo Jr, Z.H. Yeom, R.B. Robinson, M.R. Rosen, Cardiovascular research, 59, 339 (2003)

24. E. Passini, O.J. Britton, H.R. Lu, J. Rohrbacher, A.N. Hermans, D.J. Gallacher, R.J. Greig, A. Bueno-Orovio, B. Rodriguez, Frontiers in physiology, 8, 668 (2017)

25. R. Gonzalez, J. Gomis-Tena, A. Corrias, J.M. Ferrero, B. Rodriguez, J. Saiz, Sex and age related differences in drug induced $Q T$ prolongation by dofetilide under reduced repolarization reserve in simulated ventricular cells, in 2010 Annual International Conference of the IEEE Engineering in Medicine and Biology, 3245-3248 (IEEE, 2010)

26. M. Gönczi, P. Birinyi, B. Balazs, N. Szentandrássy, G. Harmati, Z. Koenczei, L. Csernoch, P.P. Nánási, Gen. Physiol. Biophys., 31, 153 (2012) 